\title{
Espumado de mezclas de silicato de sodio-wollastonita por microondas
}

\author{
G. VARGAS, F. VÁZQUEZ, J. LÓPEZ, J. MÉNDEZ, M. MÉNDEZ, P. PENA \\ CINVESTAV Unidad Saltillo, Coahuila, México. \\ 'Instituto de Cerámica y Vidrio, CSIC. Cantoblanco, Madrid, España.
}

\begin{abstract}
Se analiza el efecto de la aplicación de las microondas durante 1,5 minutos sobre el cambio de volumen, porosidad y conductividad térmica de probetas de silicato de sodio/wollastonita. Se han estudiado mezclas con 20 a $100 \%$ de silicato de sodio ${ }^{1}$. Se obtuvo una pérdida en masa del $19 \%$ y un incremento de volumen del $50 \%$ en mezclas con $20 \%$ de silicato de sodio. A medida que el contenido de éste aumentó, la pérdida en masa se incrementó hasta $27 \%$ y el cambio de volumen hasta en $500 \%$ para probetas con $100 \%$ de silicato de sodio. Se obtuvieron espumas cerámicas con porosidades totales de $65 \%$ en probetas con $20 \%$ de silicato de sodio (densidades aparente de $1,0 \mathrm{y}$ real de 2,75 g/cc) y de $91 \%$ en muestras con $100 \%$ de silicato de sodio (densidades aparente de 0,182 y real de 2,0 g/ cc). El tamaño de poro más uniforme se obtuvo para contenidos del $80 \%$ de silicato de sodio $(10$ a $30 \mu \mathrm{m})$. Las conductividades térmicas de las espumas cerámicas fueron de $0,2,0,125$ y $0,065 \mathrm{~W} / \mathrm{m} \cdot \mathrm{K}$ para contenidos de 40,70 y $100 \%$ de silicato de sodio, respectivamente.
\end{abstract}

Palabras clave: Espumas cerámicas, microondas, silicatos

\section{Foaming of sodium silicate-wollastonite mixtures by microwaves}

The effect of the application of microwaves on the volume, porosity and thermal conductivity of sodium silicate-wollastonite mixtures was studied. The compositional range studied was 20-100 wt $\%$ sodium silicate. A weight loss of $19 \%$ and a volume increase of 50\% were obtained for mixtures with $20 \mathrm{wt} \%$ sodium silicate. The weight loss increased up to $27 \%$ with increasing sodium silicate content. Volume increased in $500 \%$ for samples with $100 \mathrm{wt} \%$ sodium silicate. Ceramic foams with total porosities of $65 \mathrm{vol} \%$ were obtained for specimens containing 20 wt $\%$ sodium silicate (with apparent and real densities of 1.0 and $2.75 \mathrm{~g} / \mathrm{cc}$, respectively), while samples containing $100 \mathrm{wt} \%$ sodium silicate presented total porosities of $91 \mathrm{vol} \%$ (with apparent and real densities of 0.182 and $2.0 \mathrm{~g} / \mathrm{cc}$, respectively). The best pore size uniformity (10-30 $\mu \mathrm{m}$ ) was obtained for a sodium silicate content of $80 \mathrm{wt} \%$. The thermal conductivities of the ceramic foams were $0.2,0,125 \mathrm{and} 0,065 \mathrm{~W} / \mathrm{m} \cdot \mathrm{K}$ for sodium silicate contents of 40, 70 and $100 \mathrm{wt} \%$, respectively.

Key words: Ceramic foams, microwaves, silicates.

\section{INTRODUCCIÓN}

Existen diferentes métodos para generar cuerpos porosos [1-7]. Entre ellos se encuentran los que utilizan mezclas de materiales cerámicos con materiales combustibles que dejan huecos en la matriz cerámica al ser eliminados mediante tratamiento térmico. Un segundo grupo se basa en atrapar en un medio viscoso burbujas de gas generadas por la descomposición de alguna materia prima durante el tratamiento térmico. Otro grupo utiliza reacciones químicas que liberan gas en un medio líquido. En este caso, si se controla la viscosidad de la suspensión, es posible atrapar las burbujas y obtener un compacto poroso.

Una alternativa fácilmente aplicable y económica es el espumado térmico de silicatos alcalinos. Cuando ciertas mezclas de materiales cerámicos y silicato de sodio son sometidas a un proceso térmico adecuado, se produce la deshidratación de este último liberando vapor de agua, el cual a su vez genera la formación de cuerpos porosos [1]. Generalmente en las formulaciones de silicato de sodio se incluyen materiales de relleno o "fillers" y un agente de curado. El agente de curado afecta la densidad final del producto [2]. Por otra parte, la función de los "fillers" es reaccionar con el silicato de sodio para producir un material insoluble en agua. Batdorf [3] establece que los materiales que contienen metales divalentes del grupo II-A de la Tabla periódica, especialmente calcio y magnesio, son los que presentan mayor reactividad con los silicatos metálicos. Los materiales espumados obtenidos a partir de silicatos de sodio muestran en general buenas propiedades de aislamiento térmico. Las espumas producidas por Temple [2] presentaron una conductividad térmica entre 0,033-0,045
$\mathrm{W} / \mathrm{m} \cdot \mathrm{K}$. En este trabajo, el silicato de sodio se mezcló con silicato de calcio, silicato de magnesio, cemento portland y sílice. La temperatura de espumado fue de $700 \mathrm{~K}$. Por otra parte, los productos obtenidos por Cisneros [5] presentaron una conductividad térmica de 0,0677 $\mathrm{W} / \mathrm{m} \cdot \mathrm{K}$. En este caso, se obtuvo un producto poroso a partir de silicato de sodio expandido, el cual posteriormente fue mezclado con silicato de sodio líquido, caolín, mica y fibra inorgánica. La mezcla resultante fue prensada y sometida a un proceso de curado y secado a una temperatura entre 390 y $402 \mathrm{~K}$. En el presente trabajo se analiza el efecto de la aplicación de las microondas sobre el cambio de volumen, porosidad y conductividad térmica de probetas de silicato de sodio/ wollastonita. En un trabajo previo [8], se describió el efecto del calentamiento por microondas sobre el espumado de mezclas cerámicas a base de silicatos de sodio.

\section{PROCEDIMIENTO EXPERIMENTAL}

\subsection{Materias primas.}

Silicato de sodio: El silicato de sodio utilizado es un polvo con un tamaño de partícula $<150 \mu \mathrm{m}$ obtenido al secar por asperjado una solución de silicato de sodio. Este material es producido industrialmente por Vitro PQ Química y presenta una relación $\mathrm{SiO}_{2} / \mathrm{Na}_{2} \mathrm{O}$ en masa de 3,22 , conteniendo $19,91 \% \mathrm{Na}_{2} \mathrm{O}, 64,09 \% \mathrm{SiO}_{2}$ y $16 \% \mathrm{H}_{2} \mathrm{O}$. 
Wollastonita: El silicato de calcio utilizado en el presente estudio es producido industrialmente por la compañía NYCO, con un tamaño de partícula $<75 \mu \mathrm{m}$. Los resultados del análisis químico utilizando la técnica de fluorescencia de rayos $\mathrm{X}$, indicaron la siguiente composición para la wollastonita: $0,25 \% \mathrm{Fe}_{2} \mathrm{O}_{3^{\prime}} 0,02 \% \mathrm{TiO}_{2^{\prime}}, 46 \% \mathrm{CaO}, 0,3 \% \mathrm{~K}_{2} \mathrm{O}, 51 \%$ $\mathrm{SiO}_{2^{\prime}}, 0,66 \% \mathrm{Al}_{2} \mathrm{O}_{3^{\prime}} 0,57 \% \mathrm{Na}_{2} \mathrm{O}$ y $0,5 \% \mathrm{MgO}$. El área superficial específica de la wollastonita fue de $1,21 \mathrm{~m}^{2} / \mathrm{g}$ [determinada por el método de adsorción de nitrógeno (BET), en un equipo Gemini 2360 de Micromeritics]. La distribución de tamaño de partícula de la wollastonita se determinó por difracción de rayos láser en un equipo Coulter. El intervalo de tamaño de partícula fue de 0,4-100 $\mu \mathrm{m}$, con un tamaño promedio de $18 \mu \mathrm{m}$.

\subsection{Formulaciones estudiadas.}

Las formulaciones wollastonita/silicato de sodio estudiadas en este trabajo se indican en la Tabla I.

TABLA I. COMPOSICIONES DEL SISTEMA ESTUDIADO.

\begin{tabular}{|c|c|c|c|c|}
\hline \% Silicato de sodio & $\%$ de wollastonita & $\% \mathrm{Na}_{2} \mathrm{O}$ & $\% \mathrm{SiO}_{2}$ & $\% \mathrm{CaO}$ \\
\hline 100 & 0 & 23.7 & 76.3 & 0 \\
\hline 90 & 10 & 21 & 73.5 & 5.6 \\
\hline 80 & 20 & 18.2 & 70.83 & 10.92 \\
\hline 70 & 30 & 13.44 & 58.56 & 13.8 \\
\hline 60 & 40 & 13.18 & 65.77 & 21.05 \\
\hline 40 & 60 & 8.47 & 61.06 & 30.46 \\
\hline 20 & 80 & 4.09 & 56.67 & 39.23 \\
\hline
\end{tabular}

\subsection{Preparación de las espumas cerámicas}

La preparación de probetas incluyó las siguientes etapas: homogeneización de los polvos, prensado, humectado y esponjado térmico.

Homogeneización de polvos. Esta operación fue efectuada en un molino de bolas de 3,5 litros de capacidad usando como medio de molienda bolas de alúmina de 3 tamaños diferentes, las cuales ocuparon aproximadamente el $50 \%$ de dicho volumen. El tiempo de tratamiento fue de $10 \mathrm{~min}$ a $250 \mathrm{rpm}$.

Humectado. La cantidad de agua agregada para humectar el polvo antes de ser prensado fue de $22 \%$.

Prensado de polvos. El prensado se efectuó en una prensa Behuler con capacidad de $20 \mathrm{MPa}$. La presión para elaborar las pastillas fue de $10 \mathrm{MPa}$.

Esponjado en mufla eléctrica de resistencias. El esponjado de las pastillas se efectuó en una mufla a una temperatura de $525 \mathrm{~K}$ por 3 horas.

Esponjado en horno de microondas. Las pruebas de espumado fueron efectuadas en un horno de microondas CEM modelo Mars 5, de 1200 watts. El tiempo de procesamiento fue de 90 segundos. La temperatura promedio alcanzada en el centro de las muestras en todas las pruebas fue aproximadamente de $90^{\circ} \mathrm{C}$.

\subsection{Caracterización de los cuerpos porosos}

Densidad. La densidad real del sólido se determinó utilizando un picnómetro de helio de la marca Quantachrome.

Conductividad térmica. Estas mediciones se efectuaron a temperatura ambiente $\left(23^{\circ} \mathrm{C}\right)$ utilizando un equipo Ctmetre. Para esta determinación fue necesario hacer placas de las esponjas de aproximadamente $10 \mathrm{~cm}$ de largo por $4 \mathrm{~cm}$ de ancho y $2 \mathrm{~cm}$ de espesor. Las muestras fueron esponjadas después de dejarlas reposar por 5 horas.

Morfología y tamaño de los poros. Las espumas obtenidas se han estudiado por microscopia electrónica de barrido (MEB) utilizando un equipo Jeol JSM 6300.

\section{RESULTADOS}

\subsection{Esponjado en horno eléctrico convencional.}

El calentamiento de las probetas en un horno eléctrico de resistencias originó piezas con poros muy grandes, como se muestra en la Figura 1. Cuando el calentamiento es de afuera hacia adentro, primero se reblandece la superficie de la pastilla, generándose una capa viscosa en esta sección. Conforme se va calentando la parte interna de dicha pastilla, la superficie se vuelve cada vez más viscosa, de manera que el vapor generado en el interior es atrapado originando la aparición de poros muy grandes. Por otra parte, los poros que pudieron haberse formado en el interior se colapsan por la presión de vapor acumulada.

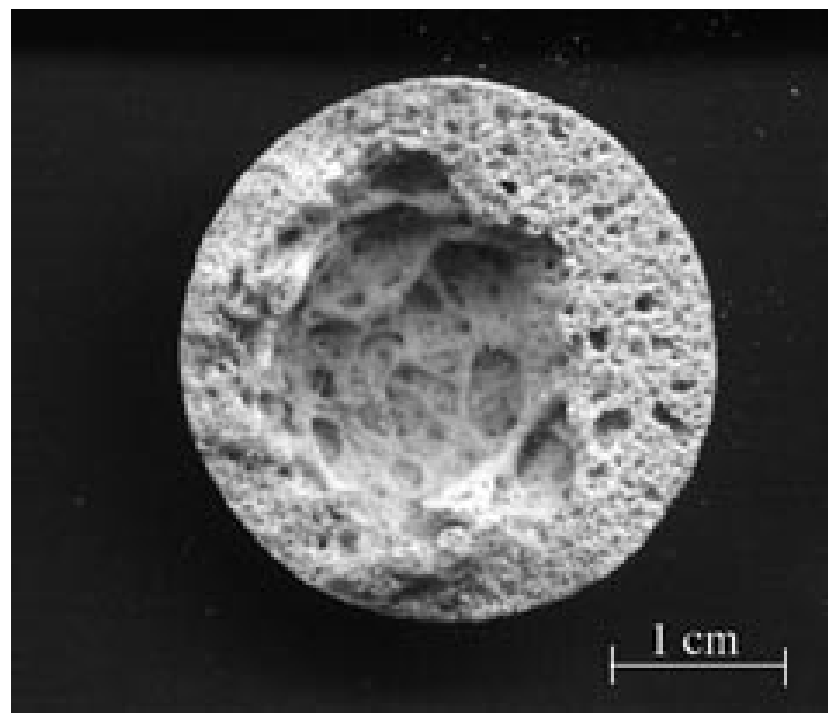

Figura 1. Fotografía obtenida por microscopia estereográfica, mostrando la textura del material poroso obtenido por esponjado en un horno eléctrico de resistencias de la composición $80 \%$ silicato de sodio / $20 \%$ wollastonita.

\subsection{Esponjado en un horno de microondas.}

Mediante el calentamiento por microondas se obtuvieron esponjas cerámicas con una distribución de tamaño de poro muy uniforme, como muestra la Figura 2.

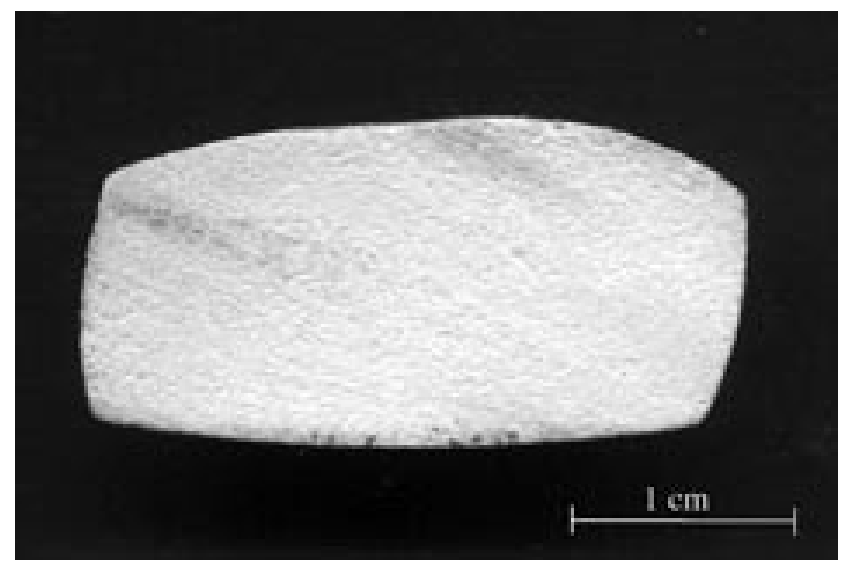

Figura 2. Fotografía obtenida por microscopía estereográfica de un corte transversal de una esponja obtenida mediante calentamiento por microondas, composición $80 \%$ silicato de sodio $/ 20 \%$ wollastonita. 

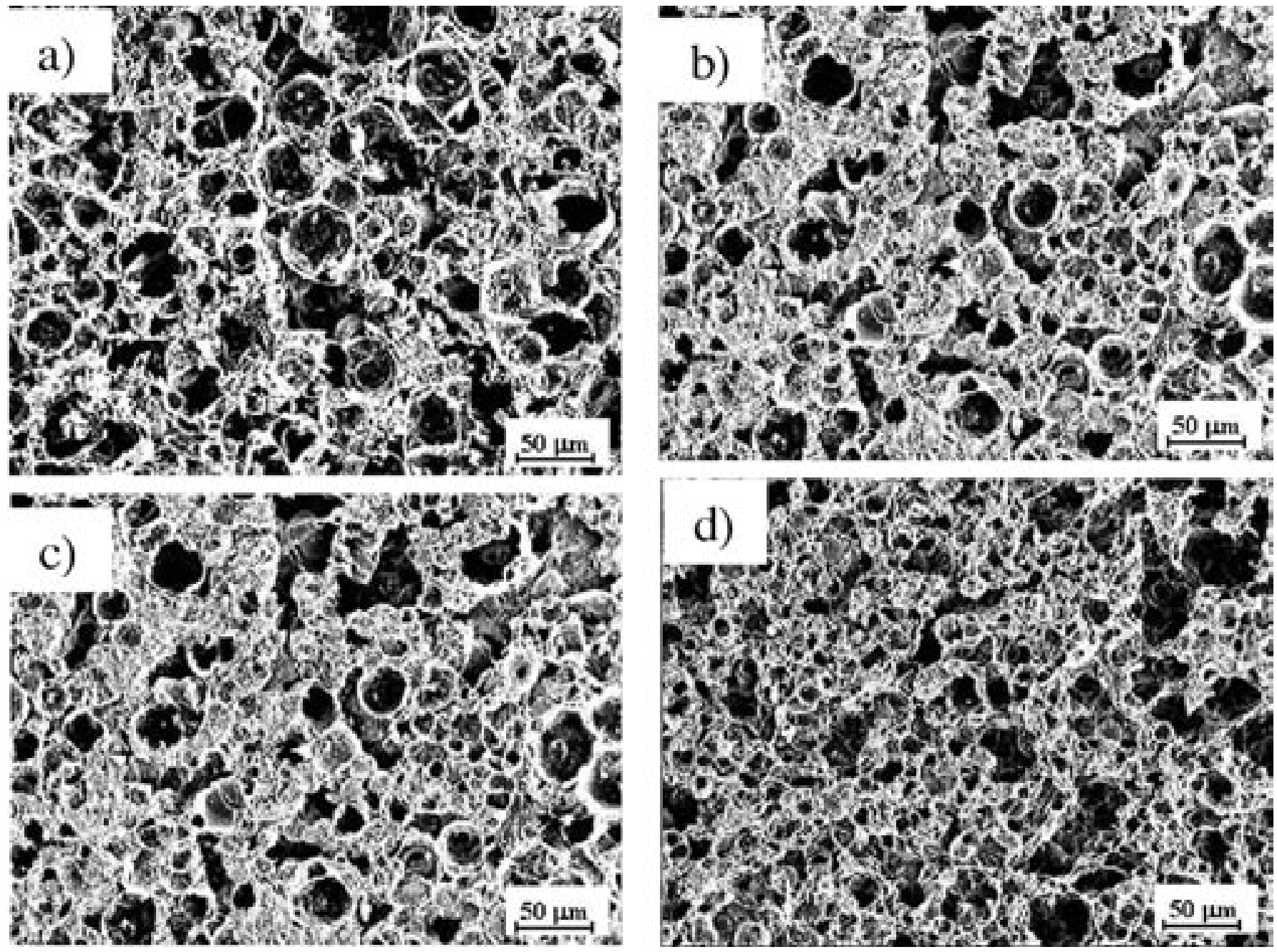

Figura 3. Fotomicrografias tomadas por MEB de esponjas cerámicas obtenidas por calentamiento de microondas de mezclas de silicato de sodio / wollastonita, con las proporciones de silicato de sodio siguientes: a) $100 \%$, b) $90 \%$, c) $80 \%$ y d) $70 \%$.

El tamaño de poro según los resultados obtenidos por MEB varia con la composición y la temperatura, obteniéndose las distribuciones mas uniformes en la muestra con el $80 \%$ de silicato de sodio con valores entre 10-30 $\mu \mathrm{m}$. Como se aprecia en las fotomicrografias obtenidas por MEB mostradas en la Figura 3, se ha observado que al aumentar el contenido de wollastonita disminuye el tamaño de los poros.

El proceso de espumado se inició dentro de los primeros 10 segundos de calentamiento, lo cual corresponde a una temperatura de aproximadamente $325 \mathrm{~K}$. En el caso del calentamiento por microondas [8], el mecanismo de esponjado es diferente. Aquí el calentamiento se inicia en el interior de la pieza, por lo tanto, es ahí donde primeramente se produce vapor. Dicho vapor encontrará resistencia a su escape, en virtud de la capa de silicato de sodio disuelto que se encuentra en la superficie de las partículas. Esto origina que el vapor empiece a reblandecer todas las partículas y finalmente la presión de vapor generada producirá el esponjamiento.

En la Figura 4 se muestra el porcentaje de expansión, así como el porcentaje de pérdida en masa, que experimentaron las pastillas al ser esponjadas. Las pastillas sufrieron una expansión mayor conforme se incrementó el contenido de silicato de sodio en la formulación. Como se puede observar, la composición con 100\% de silicato de sodio experimentó la mayor pérdida en peso, lo que generó una mayor cantidad de vapor, dando como resultado que la esponja con esta formulación haya mostrado un volumen total mayor comparado con el resto de las esponjas.

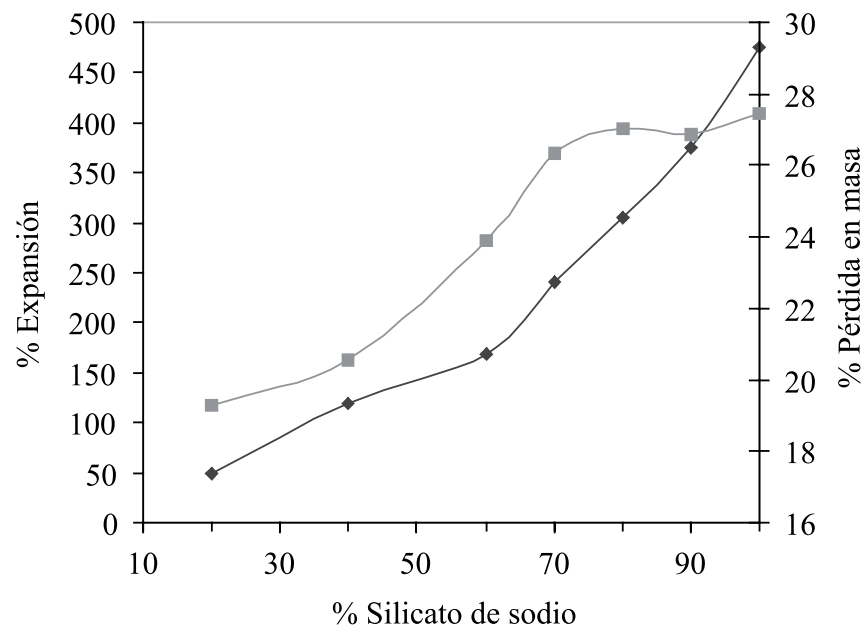

Figura 4. Porcentaje de expansión $(\diamond)$ y de pérdida en masa (प) de las pastillas.

Con la finalidad de cuantificar la porosidad total en volumen, se procedió a determinar la densidad real del sólido para cada esponja, lo cual permitió calcular el volumen ocupado por la masa de dicha esponja cuando no existe porosidad. Posteriormente, conociendo el volumen total de la esponja fue posible calcular el volumen ocupado por los poros y la porosidad total. 


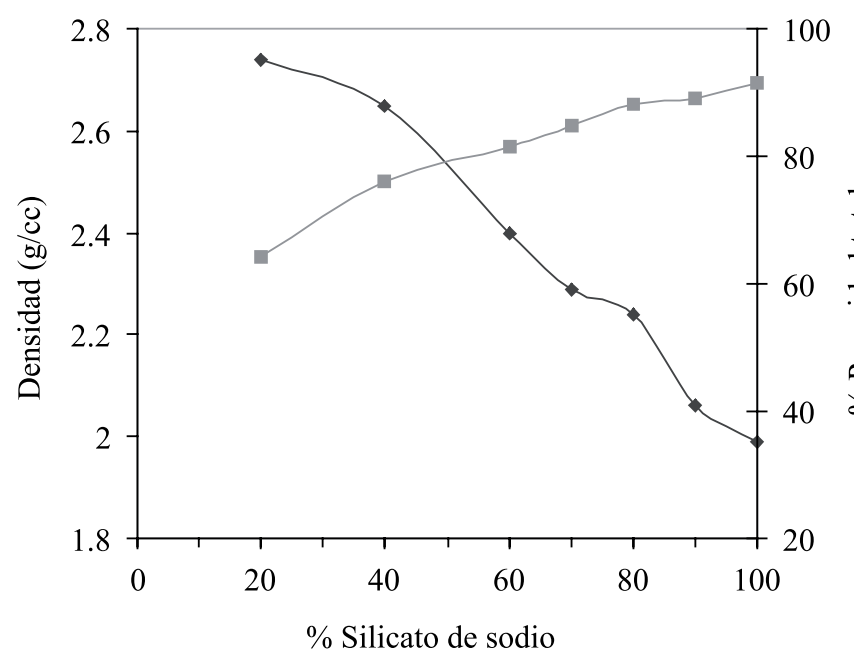

Figura 5. Densidad real $(\bullet)$ y porosidad total en volumen () de las esponjas.

En la Figura 5 se muestra como la densidad real disminuye desde $2.75 \mathrm{~g} / \mathrm{cc}$ en esponjas con $20 \%$ de silicato de sodio hasta un valor de 2 $\mathrm{g} / \mathrm{cc}$ para probetas con $100 \%$ de silicato de sodio.

En la Figura 5 se muestra también la porosidad total en volumen para cada esponja. La mayor porosidad $(90 \%)$ fue presentada por las esponjas con $100 \%$ de silicato de sodio. Conforme disminuyó el contenido de silicato de sodio, la porosidad disminuyó hasta un $65 \%$ para muestras con $20 \%$ de silicato de sodio.

\subsection{Conductividad térmica.}

Una vez comprobado que el espumado por microondas es un procedimiento adecuado para preparar esponjas cerámicas con elevada porosidad y buena homogeneidad se determinó la conductividad térmica de las composiciones conteniendo 100, 70 y 40\% de silicato de sodio Figura 6.

La menor conductividad térmica $(0,065 \mathrm{~W} / \mathrm{m} \cdot \mathrm{K})$ fue obtenida en esponjas con $100 \%$ de silicato de sodio. Este valor es muy cercano a los valores de $0,033-0,045 \mathrm{~W} / \mathrm{m} \cdot \mathrm{K}$ obtenidos por Temple [2] y al valor de $0,0677 \mathrm{~W} / \mathrm{m} \cdot \mathrm{K}$ reportado por Cisneros [5], para la conductividad térmica de materiales expandidos a base de silicato de sodio. A medida que se incrementó la cantidad de wollastonita, la conductividad térmica se incrementó hasta $0,2 \mathrm{~W} / \mathrm{m} \cdot \mathrm{K}$ para una mezcla de $60 \%$ de wollastonita. La conductividad térmica depende fuertemente de la porosidad y de la composición mineralógica, por lo cual la disminución en la conductividad térmica observada al incrementar el contenido de silicato de sodio puede ser atribuida a estos dos factores.

\section{CONCLUSIONES}

El esponjado en una mufla da como resultado cuerpos con poros grandes e irregulares en su interior. Los cuerpos porosos obtenidos en un horno de microondas son más uniformes y tienden a conservar

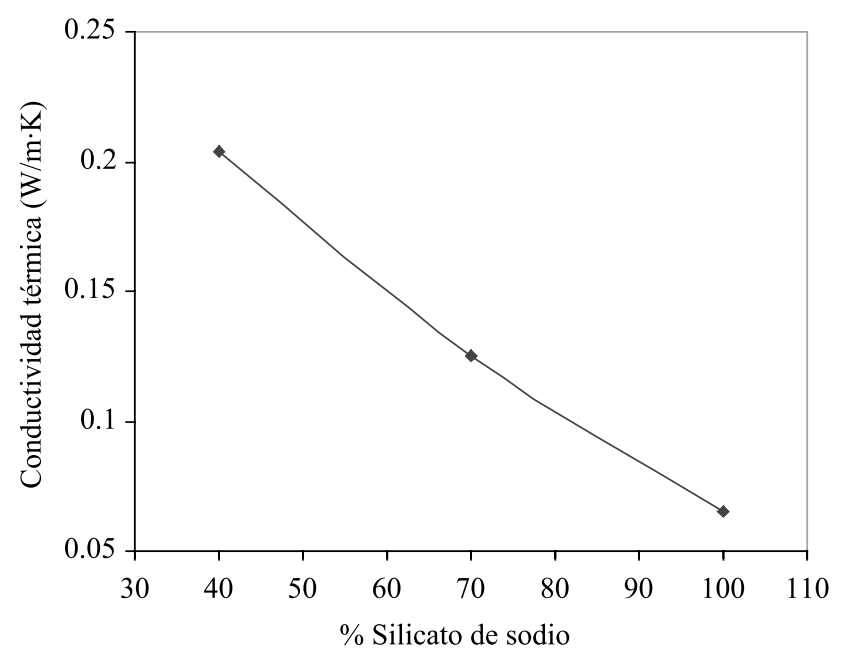

Figura 6. Conductividad térmica de las esponjas $\left(23^{\circ} \mathrm{C}\right)$.

la forma geométrica inicial al final del calentamiento por microondas. El inicio del esponjamiento en un horno de microondas ocurre en los primeros 10 segundos de calentamiento, lo cual corresponde a una temperatura de aproximadamente $325 \mathrm{~K}$. Mediante el método desarrollado en este trabajo es posible obtener cuerpos porosos a base de silicato de sodio y wollastonita a través de una expansión térmica en un horno de microondas con tiempos de procesamiento de 90 segundos. De acuerdo a la relación silicato de sodio/silicato de calcio empleada, dichos cuerpos porosos muestran densidades aparentes desde 0,182 hasta $0,996 \mathrm{~g} / \mathrm{cc}$, porosidades totales en volumen desde $45 \%$ hasta $91 \%$ y conductividades térmicas desde $0,065 \mathrm{~W} / \mathrm{m} \cdot \mathrm{K}$ hasta $0,203 \mathrm{~W} / \mathrm{m} \cdot \mathrm{K}$. A mayor contenido de silicato de sodio en las esponjas, se tiene una menor densidad aparente, mayor porosidad y menor conductividad térmica.

\section{BIBLIOGRAFÍA}

1. Temple et al, "Light weight silicate aggregate", US Patent No. 4,396,723, 1983.

2. Temple et al, "Light weight silicate aggregate", US Patent No. 4,203,773, 1980.

3. Banks et al, "High strength water resistant silicate foam", US Patent No. 3,933,514, 1976

4. Batdorf, "Curable aqueous silicate composition, uses thereof, and coatings or layers made there from", US Patent No. 4,347,285, 1982.

5. Cisneros, "Thermal insulating material and methods of production thereof", US Patent No. 4,664,712, 1987.

6. Popov et al, "Water resistant and heat insulating material and method of making same", US Patent No. 4,451,294, 1984.

7. Shepherd et al, "Light weight building material board", US Patent No. $5,256,222$

8. G. Vargas, J. C. Pérez, J. Méndez, M. Méndez y P. Pena, “Espumado por microondas de mezclas de silicato de sodio-ortofosfato cálcico dibásico", Bol. Soc. Esp. Ceram. V., 41 [5] 481-486 (2002).

Recibido: 01.02 .03

Aceptado: 30.11 .03 